\title{
Carriage of Methicillin-Resistant STAPHYLOCOCCUS AUREUS AT HOSPITAL ADMISSION
}

\author{
Nicolas Troillet, MD, MSc; Yehuda Carmeli, MD; Matthew H. Samore, MD; Joanna Dakos, BS, MT (ASCP);
} Karen Eichelberger, BS, MT (ASCP); Paola C. DeGirolami, MD; Adolf W. Karchmer, MD

\begin{abstract}
OBJECTIVES: To measure the prevalence of, and to establish predictors for, the nasal carriage of methicillinresistant Staphylococcus aureus (MRSA) at hospital admission. To evaluate mannitol-salt agar with oxacillin for the simultaneous detection and identification of MRSA from nasal swabs.

DESIGN: Three-month prospective case-control survey, with data collected from interviews and computerized databases. The criterion standard for MRSA detection was culture on Mueller-Hinton agar with oxacillin $6 \mu \mathrm{g} / \mathrm{mL}$ (National Committee for Clinical Laboratory Standards method).

SETTING: 320-bed tertiary-care hospital.

PATIENTS: 387 patients screened within 24 hours after admission, including 10 MRSA carriers (cases), 291 patients with no $S$ aureus, and 86 patients with methicillinsusceptible $S$ aureus.

RESULTS: The prevalence of MRSA nasal carriage

was $2.6 \%$, whereas the prevalence of carriage was $3.1 \%$ when both nasal and wound cultures were performed. The significant predictors of carriage were a prior detection of MRSA, open wounds, diabetes mellitus, treatments by injection, prior nursing home stays, visits at home by a nurse, and prior antibiotic treatments. Cases had stayed for longer periods in hospitals and had received longer antibiotic treatments within a year. Eighty patients (including the 10 cases) had diabetes, had been exposed to healthcare facilities within a year, and had antibiotics within 6 months. The sensitivity and negative predictive value of nasal swabs on mannitol-salt agar with oxacillin were $60 \%$ and $71 \%$, respectively.

CONCLUSION: MRSA carriage on admission to the hospital may be an increasing and underestimated problem. Further studies are needed to develop and validate a sensitive and specific prediction rule (Infect Control Hosp Epidemiol 1998;19:181-185).
\end{abstract}

The threat of methicillin-resistant Staphylococcus aureus (MRSA) in hospitals was recognized first in Europe and the United States in the late 1960s. 1,2 Nowadays, MRSA has been detected worldwide and accounts for a growing proportion of $S$ aureus isolated from hospitalized patients in many countries. ${ }^{3-5}$

Mulligan and colleagues, in their comprehensive review, remind us that "there are two major mechanisms for the introduction of MRSA into an institution: the most common is the admission of an infected or colonized patient who serves as a reservoir; less frequent is the arrival of a colonized or infected healthcare worker who disseminates the organism directly". 6

Until recently, index cases were thought to have acquired MRSA in another hospital or a nursing

home, with the exception of rare communityacquired outbreaks that occurred mainly among intravenous drug users. ${ }^{7}$ The described risk factors for the nosocomial acquisition of MRSA are a long hospital stay, numerous or prolonged antibiotic courses, the presence of invasive devices, or proximity to an already infected or colonized inpatient. ${ }^{8}$

Since then, and secondary to recent trends in health care, concerns arose about the potential spread of MRSA to the community. ${ }^{9}$ In three recent reports on the epidemiology of MRSA at institutions in Canada, Switzerland, and the United States, 20\% to 62\% of patients colonized with this organism were detected within 48 to 72 hours after admission. ${ }^{10-12}$ A substantial proportion of these patients presented no known risk factors for MRSA carriage. However, these stud-

From the Division of Infectious Diseases (Drs. Troillet, Carmeli, Samore, and Karchmer), and the Department of Pathology (Ms. Dakos, Ms. Eichelberg, and Dr. DeGirolami), Beth Israel Deaconess Medical Center, Harvard Medical School, Boston, Massachusetts. Address reprint requests to Nicolas Troillet, MD, Division of Infectious Diseases, Central Institute of the Valais Hospitals, 86 Avenue Grand-Champsec, CH-1951 Sion, Switzerland.

97-OA-043. Troillet N, Carmeli Y, Samore MH, Dakos J, Eichelberg K, DeGirolami PC, Karchmer AW. Carriage of methicillinresistant Staphylococcus aureus at hospital admission. Infect Control Hosp Epidemiol 1998;19:181-185. 
ies were descriptive and did not explore systematically past exposures to the healthcare system.

We designed this study to determine the prevalence of MRSA carriage on admission to our hospital and to establish predictors for such a carriage. A secondary goal was to evaluate mannitol-salt agar with oxacillin $4 \mu \mathrm{g} / \mathrm{mL}$ for the simultaneous detection and identification of MRSA from nasal swabs.

\section{METHODS}

The Deaconess Hospital is a 320-bed, tertiarycare, urban, teaching hospital with approximately 10,000 admissions per year. The routine surveillance for MRSA at this institution is laboratory-based. In $1995,18 \%(201 / 1,116)$ of the patients with clinical cultures positive for $S$ aureus were colonized or infected with MRSA, of whom 113 (56\%) were new or incident cases. Only 43 of the latter (38\%) were considered as nosocomial. The remaining $62 \%$ were detected within the first 72 hours of their hospital stay or were outpatients.

After approval by the Institutional Review Board, surveillance cultures were performed, in a nonepidemic setting, from September 25 to December 23, 1996, on two wards chosen to reflect a large proportion of our hospital's activities. Ward A admits mostly patients for vascular surgery and podiatry; ward B admits patients for general medicine and general surgery. Weekly, from Monday through Thursday, patients who had been admitted within the past 24 hours and had not been identified already as MRSA carriers within this study were asked to participate.

Nasal cultures were collected from every consenting patient by using moistened rayon swabs (Starswab; Starplex Scientific, Gormley, Ontario, Canada) rotated five times in both anterior nares. The samples were carried within 2 hours to the laboratory and processed according to a method adapted from Lally and colleagues. ${ }^{13}$ Briefly, swabs were kept overnight at $35^{\circ} \mathrm{C}$ in an enrichment tryptic soy broth with $7.5 \% \mathrm{NaCl}$ and later plated onto mannitol-salt agar with and without $4 \mu \mathrm{g} / \mathrm{mL}$ of oxacillin (Remel, Lenexa, KS). The plates (two per patient) were incubated at $35^{\circ} \mathrm{C}$ with $5 \% \mathrm{CO}_{2}$ for 24 hours. Patients with mannitol-fermenting, coagulase-positive colonies growing only on the plate without oxacillin were considered carriers of methicillin-susceptible $S$ aureus (MSSA). Those with growth of such colonies on both plates were considered MRSA carriers. All $S$ aureus isolates subsequently were screened for methicillin resistance on Mueller-Hinton agar supplemented with salt and oxacillin $6 \mu \mathrm{g} / \mathrm{mL}$, according to National Committee for Clinical Laboratory Standards
(NCCLS) guidelines. ${ }^{14}$ The sensitivity, specificity, and negative predictive value of mannitol-salt agar with oxacillin were calculated, the NCCLS method being considered the criterion standard.

Wound swabs, when available, were processed routinely by the laboratory and plated directly onto sheep blood agar. $S$ aureus growing from these cultures were screened for methicillin resistance according to the NCCLS guidelines, as above. ${ }^{14}$

A case-control study was implemented to establish predictors for the nasal carriage of MRSA on admission. Cases were patients with nasal cultures positive for MRSA. A first control group consisted of patients with no $S$ aureus isolated from their nares. Patients with nares cultures that yielded only MSSA constituted a second control group.

Initially, interviews were administered after cases and controls had been identified. From October 16, all patients were asked five questions at the time the nasal swabs were collected. They were queried regarding the presence of an open wound, the time elapsed since their last hospital stay, last nursing home stay, last antibiotic treatment, and last outpatient visit to a hospital. Additional information collected by written questionnaires included the presence of invasive devices on admission, ambulatory dialysis, treatments by means of injections, and community exposures to healthcare workers or other persons at risk for MRSA carriage. Most exposures were considered for as far back as 5 years. Information on underlying diseases was collected from the hospital administrative database by using Access (Microsoft, Redmond, WA). Comorbidities were weighted according to the Charlson score. 15 Variables that were associated significantly with MRSA carriage were used to develop a preliminary prediction rule.

Data were entered in Epi Info (Centers for Disease Control and Prevention, Atlanta, GA, and World Health Organization, Geneva, Switzerland) and analyzed in SAS (SAS Institute, Cary, NC). Differences in proportions and means between cases and controls were tested by Fisher's Exact Tests, twosample $t$ tests, or Wilcoxon Rank Sum Tests, as appropriate. All tests were two-tailed; $P \leqslant .05$ was considered significant. To calculate a limiting odds ratio $(\mathrm{OR})$ in cases where the numerator of a given proportion (rate) either was zero or equalled the denominator (which would result in an empty cell in a two-by-two table, preventing calculation of the OR), the calculation was performed based on the next less extreme distribution, in which the numerator equalled one, or one less than the denominator, respectively, and the result is shown as greater than (or less than, if appropriate) the OR calculated thereby. 


\section{RESULTS}

There were 746 eligible admissions to wards A and B during the study period, of whom 412 were asked for consent, and 387 agreed to participate in the study. Mean age was 58.2 years (range, 17-93); 164 were females and 223 males.

$S$ aureus was recovered from nasal swabs taken from 96 patients (24.8\%), of whom 6 were colonized by MRSA as determined on mannitol salt screening. However, subsequent evaluation of the 96 isolates on Mueller-Hinton media supplemented with $\mathrm{NaCl}$ and oxacillin $6 \mu \mathrm{g} / \mathrm{mL}$ (NCCLS method) disclosed 4 additional MRSA that previously had been misidentified as MSSA. Thus, the prevalence of MRSA and MSSA nasal carriage on admission was 10 of 387 (2.6\%) and 86 of $387(22.2 \%)$, respectively.

When the NCCLS method was considered the gold standard, the sensitivity of mannitol-salt agar with oxacillin was $60 \%$, its specificity was $100 \%$, and its negative predictive value was $71 \%$ (Table 1).

Of the 387 admissions, 314 were queried for the presence of an open wound. Of 95 with an open wound, 71 had cultures obtained within 24 hours after hospital admission. These 71 wound cultures revealed no $S$ aureus in 43 (60.6\%), MSSA in 22 (31\%), and MRSA in 6 (8.4\%), of whom 5 also had nasal cultures positive for MRSA and 1 had no $S$ aureus in the nares. Thus, of 290 patients with either no open wound or wounds from which cultures were obtained, 9 (3.1\%) carried MRSA at the time of hospital admission in their nares, a wound, or at both sites.

To define the features of patients who carry MRSA, the 10 patients from whom nasal swab cultures yielded MRSA (cases) were compared to the 291 patients with no $S$ aureus isolated from nares and to the 86 patients with MSSA isolated from nares. The significant differences between these groups are given in Table 2. Cases were more likely than patients in either control group to have been detected previously as MRSA carriers and to suffer from an open wound and from diabetes. They also had been hospitalized for longer periods of time during the past year and were more likely to have been residents in chronic-care facilities and visited by a healthcare worker at home within the past year. Cases also were more likely to have received antibiotics within the past 6 months and to have received them for longer periods. They were more likely than patients with MSSA to be treated by means of injections. No significant differences were found between cases and either control group for age, gender, various underlying conditions other than diabetes or the presence of an open wound, Charlson comorbidity score, presence of invasive devices, undergoing dialysis, being
TABLE 1

A Comparison of Mannitol-Salt Agar With OXACILlin $4 \mu \mathrm{G} / \mathrm{ML}$ VERSUS MUELlER-Hinton With SALT AND OXACILLIN $6 \mu \mathrm{G} / \mathrm{ML}$ (NCCLS METHOD) FOR THE Detection of Methicillin-RESistant STAPHYLOCOCCUS AUREUS

\begin{tabular}{lcccc}
\hline & & \multicolumn{2}{c}{ Mannitol-Salt } & \\
\cline { 3 - 4 } & & MRSA & MSSA & \\
\hline \multirow{2}{*}{ NCCLS method } & MRSA & 6 & 4 & 10 \\
\multirow{2}{*}{ Total } & MSSA & 0 & 86 & 86 \\
& & 6 & 90 & 96 \\
\hline
\end{tabular}

Abbreviations: MRSA, methicillin-resistant Staphylococcus aureus; MSSA, methicillin-susceptible $S$ aureus; NCCLS, National Committee for Clinical Laboratory Standards

$P=.045$ (McNemar's Test).

transferred from a hospital or a nursing home, or having visited the outpatient department of a hospital. The small number of outcomes, missing values, and "empty cells" prevented the use of multivariate logistic regression to adjust for confounding.

Methicillin-resistant $S$ aureus carriage in 4 of the 10 cases had been detected during past admissions, 12 days to 23 months previously, by our routine laboratory-based surveillance system of clinical cultures. Three others who had been transferred from hospitals or nursing homes were detected early in this admission by the same surveillance system because routine wound cultures were ordered by clinicians. This laboratory-based system detected two patients much (24 and 90 days) later during their hospitalization. One patient would not have been detected at all in the absence of this study. Thus, three of the six previously unknown MRSA carriers would not have been detected on admission in the absence of surveillance cultures. All three were admitted from the community.

The three features of diabetes, antibiotic treatment within 6 months, and exposure to a healthcare facility within the past year were found in 80 (20.7\%) of the 387 study patients. All 10 MRSA carriers were in this subgroup. Forty-five patients (11.6\%) presented with the same features plus an open wound, and eight of the cases were among them. Thus, the first set of features would have a sensitivity of $100 \%$ and a specificity of $81 \%$ to detect MRSA carriage. Adding the fourth feature to this set would result in a sensitivity of $80 \%$ and a specificity of $90 \%$.

\section{DISCUSSION}

This study revealed a prevalence of $2.6 \%$ and $3.1 \%$ for MRSA carriage among patients admitted to an urban tertiary-care hospital, as determined by 
either nasal cultures alone or the combination of nasal and wound cultures, respectively. In a previous study, the sensitivity and negative predictive value of the combination of cultures from these two sites were $100 \%{ }^{16}$ In addition, the use of enrichment broth, which enhances the sensitivity of detection of $S$ aureus from nasal swabs, ${ }^{17}$ and the implementation of two different techniques to detect methicillin resistance in our isolates should have prevented most false-negative results. On the other hand, the large proportion of patients with open wounds in our population could have influenced our results. Indeed, we found, as previously suggested by others, ${ }^{18}$ that MRSA was associated significantly with skin defects.

This prevalence of MRSA carriage is approximately twice that found by Walsh and colleagues approximately 10 years ago among 253 patients admitted to the Veterans' Administration Medical Center in Baltimore. ${ }^{19}$ Besides differences in methods or populations, this difference could be due to changing habits in healthcare over the past decade. Once seen as merely an epidemic nosocomial pathogen, MRSA has become endemic in most institutions and could be spreading to the community, because the barriers between both settings are less evident nowadays than they were in the past. 20

In the absence of admission surveillance, $50 \%$ of previously unknown MRSA carriers would have been missed or detected late. Thus, the continuous introduction of MRSA into tertiary-care institutions through colonized patients admitted from the community may be an important and underestimated factor in the epidemiology of nosocomial infections due to this microorganism.

Descriptive studies have mentioned the lack of evident risk factors in some inpatients from whom MRSA was detected early after admission. ${ }^{10-12} \mathrm{We}$ addressed this issue prospectively by taking a careful medical history and by designing a case-control survey. We found that MRSA carriage, in contrast to the carriage of MSSA, was significantly associated with variables reflecting contacts with the healthcare system. All of our 10 cases had exposure to an acuteor a chronic-care facility, hospitals or nursing homes, or visits to a hospital outpatient department, within the past year.

Although community acquisition of MRSA has been reported and may be an emerging threat, 21,22 this term could be overused and misleading. Our results suggest that "community carriage" is a more appropriate term, because the acquisition of MRSA still might take place primarily in healthcare institutions, with carriage persisting in the community for 
years. 16 Causal inferences, however, should be drawn from our data with caution, because the time relationships between MRSA carriage (the outcome) and exposures to the healthcare system remain unknown, and because confounding could not be excluded by adjusted analysis.

The predictors associated with MRSA carriage at the time of admission, while not establishing a causal relationship, still may be used to identify appropriate patients for selective surveillance and the early implementation of cost-effective infection control measures. Our data suggest that, in addition to patients known to have been MRSA carriers in the past, patients who suffer from diabetes, who have been exposed to a healthcare facility during the past year and have received antibiotics within the past 6 months are appropriate targets for selective cultures on admission. Cultures should include the nares and any wound. Further studies are needed, however, to develop a more precise prediction rule by using multivariate analysis. Additionally, sensitive and specific techniques that allow rapid identification of MRSA are needed.

In conclusion, the prevalence of MRSA carriage upon admission to the hospital appears to have increased during the past decade. Although most patients still may acquire MRSA in healthcare facilities, the continued community carriage of these organisms mandates additional study of this phenomenon and potentially new approaches to infection control. Finally, it is likely that similar patterns of carriage exist for other nosocomially acquired organisms, such as vancomycin-resistant enterococci and Clostridium difficile, and that analogous investigations should be focused in these areas.

\section{REFERENCES}

1. Benner EJ, Kayser FH. Growing clinical significance of methicillin-resistant Staphylococcus aureus. Lancet 1968;ii:741-744.

2. Barret FE, McGehe RF Jr, Finland M. Methicillin-resistant Staphylococcus aureus at Boston City Hospital: bacteriologic and epidemiologic observations. N Engl J Med 1968;279: 441-448.

3. Panlilio AL, Culver DH, Gaynes RP, Banerjee S, Henderson TS, Tolson JS, et al. Methicillin-resistant Staphylococcus aureus in US hospitals, 1975-1991. Infect Control Hosp Epidemiol 1992;13:582-586.

4. Voss A, Milatovic D, Wallrauch-Schwartz C, Rosdahl VT, Braveny I. Methicillin-resistant Staphylococcus aureus in Europe. Eur J Clin Microbiol Infect Dis 1994;13:50-55.

5. Riley TV, Pearman JW, Rouse IL. Changing epidemiology of methicillin-resistant Staphylococcus aureus in Western Australia. Med J Aust 1995;163:412-414.
6. Mulligan ME, Murray-Leisure KA, Ribner BS, Standiford HC, John JF, Korvick JA, et al. Methicillin-resistant Staphylococcus aureus: a consensus review of the microbiology, pathogenesis, and epidemiology with implications for prevention and management. Am J Med 1993;94:313-328.

7. Saravolatz LD, Markowitz N, Arking L, Pohold D, Fisher E. Methicillin-resistant Staphylococcus aureus. Epidemiologic observations during a community-acquired outbreak. Ann Intern Med 1982;96:11-16.

8. Thompson RL, Cabezudo I, Wenzel RP. Epidemiology of nosocomial infections caused by methicillin-resistant Staphylococcus aureus. Ann Intern Med 1982;97:309-317.

9. Rosenberg J. Methicillin-resistant Staphylococcus aureus (MRSA) in the community: who's watching? Lancet 1995;346:132-133.

10. Embil J, Ramotar K, Romance L, Alfa M, Conly J, Cronk S, et al. Methicillin-resistant Staphylococcus aureus in tertiary care institutions on the Canadian prairies, 1990-1992. Infect Control Hosp Epidemiol 1994;15:646-651.

11. Lugeon C, Blanc DS, Wenger A, Francioli P. Molecular epidemioloy of methicillin-resistant Staphylococcus aureus at a lowincidence hospital over a four-year period. Infect Control Hosp Epidemiol 1995;16:220-267.

12. Layton MC, Hierholzer WJ, Patterson JE. The evolving epidemiology of methicillin-resistant Staphylococcus aureus at a university hospital. Infect Control Hosp Epidemiol 1995;16:12-17.

13. Lally RT, Ederer MN, Woolfrey BF. Evaluation of mannitol salt agar with oxacillin as a screening medium for methicillin-resistant Staphylococcus aureus. J Clin Microbiol 1985;22:501-504.

14. National Committee for Clinical Laboratory Standards. Methods for Dilution Antimicrobial Susceptibility Tests for Bacteria that Grow Aerobically. 2nd ed. Approved standard M7-A2. Villanova, PA: National Committee for Clinical Laboratory Standards; 1990.

15. Charlson ME, Pompei P, Ales KL, McKenzie CR. A new method of classifying prognostic comorbidity in longitudinal studies: development and validation. J Chronic Dis 1987;40:373-383.

16. Sanford MD, Widmer AF, Bale MJ, Jones RN, Wenzel RP. Efficient detection and long-term persistence of the carriage of methicillin-resistance Staphylococcus aureus. Clin Infect Dis 1994;19:1123-1128.

17. Cookson B, Peters B, Webster M, Phillips I, Rahman M, Noble W. Staff carriage of epidemic methicillin-resistant Staphylococcus aureus. J Clin Microbiol 1989;27:1471-1476.

18. Maslow JN, Brecher S, Gunn J, Durbin A, Barlow MA, Arbeit $\mathrm{RD}$. Variation and persistence of methicillin-resistant Staphylococcus aureus strains among individual patients over extended periods of time. Eur J Clin Microbiol Infect Dis 1995;14:282-290.

19. Walsh TJ, Vlahov D, Hansen SL, Sonnenberg E, Khabbaz R, Gadacz T, et al. Prospective microbiologic surveillance in control of nosocomial methicillin-resistant Staphylococcus aureus. Infect Control 1987;8:7-14.

20. Decker MD, Schaffner W. The relationship between the hospital and the community. In: Bennett JV, Brackman PS, eds. Hospital Infections. 3rd ed. Boston, MA: Little, Brown and Co; 1992:221-230

21. Berman DS, Eisner W, Kreiswirth B. Community-acquired methicillin-resistant Staphylococcus aureus infection. New Engl J Med 1993;329:1896.

22. Hollis RJ, Barr JL, Doebbeling BN, Pfaller MA, Wenzel RP. Familial carriage of methicillin-resistant Staphylococcus aureus and subsequent infection in a premature neonate. Clin Infect Dis 1995;21:328-332. 